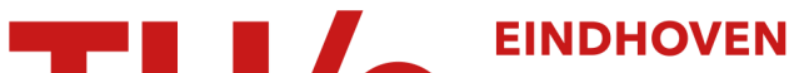 UNIVERSITY OF TECHNOLOGY
}

\section{Hydrodynamics of air entrainment by moving contact lines}

\section{Citation for published version (APA):}

Chan, T. S., Srivastava, S., Marchand, A., Andreotti, B., Biferale, L., Toschi, F., \& Snoeijer, J. H. (2013). Hydrodynamics of air entrainment by moving contact lines. Physics of Fluids, 25(7), 074105/1-19. https://doi.org/10.1063/1.4814466

DOI:

$10.1063 / 1.4814466$

Document status and date:

Published: 01/01/2013

\section{Document Version:}

Publisher's PDF, also known as Version of Record (includes final page, issue and volume numbers)

\section{Please check the document version of this publication:}

- A submitted manuscript is the version of the article upon submission and before peer-review. There can be important differences between the submitted version and the official published version of record. People interested in the research are advised to contact the author for the final version of the publication, or visit the $\mathrm{DOI}$ to the publisher's website.

- The final author version and the galley proof are versions of the publication after peer review.

- The final published version features the final layout of the paper including the volume, issue and page numbers.

Link to publication

\section{General rights}

Copyright and moral rights for the publications made accessible in the public portal are retained by the authors and/or other copyright owners and it is a condition of accessing publications that users recognise and abide by the legal requirements associated with these rights.

- Users may download and print one copy of any publication from the public portal for the purpose of private study or research.

- You may not further distribute the material or use it for any profit-making activity or commercial gain

- You may freely distribute the URL identifying the publication in the public portal.

If the publication is distributed under the terms of Article $25 \mathrm{fa}$ of the Dutch Copyright Act, indicated by the "Taverne" license above, please follow below link for the End User Agreement:

www.tue.nl/taverne

\section{Take down policy}

If you believe that this document breaches copyright please contact us at:

openaccess@tue.nl

providing details and we will investigate your claim. 


\title{
Hydrodynamics of air entrainment by moving contact lines
}

\author{
T. S. Chan, ${ }^{1}$ S. Srivastava, ${ }^{2,3}$ A. Marchand, ${ }^{4}$ B. Andreotti, ${ }^{4}$ L. Biferale,${ }^{5}$ \\ F. Toschi, ${ }^{2,3,6}$ and J. H. Snoeijer ${ }^{1}$ \\ ${ }^{1}$ Physics of Fluids Group, Faculty of Science and Technology and MESA+ Institute, \\ University of Twente, 7500AE Enschede, The Netherlands \\ ${ }^{2}$ Department of Applied Physics, Eindhoven University of Technology, \\ $5600 \mathrm{MB}$ Eindhoven, The Netherlands \\ ${ }^{3}$ Department of Mathematics and Computer Science, Eindhoven University of Technology, \\ $5600 \mathrm{MB}$ Eindhoven, The Netherlands \\ ${ }^{4}$ Physique et Mécanique des Milieux Hétérogènes, UMR 7636 ESPCI-CNRS, \\ Université Paris-Diderot, 10 rue Vauquelin, 75005 Paris, France \\ ${ }^{5}$ Department of Physics and INFN, University of Tor Vergata, Via della Ricerca Scientifica 1, \\ 00133 Rome, Italy \\ ${ }^{6}$ CNR-IAC, Via dei Taurini 19, 00185 Rome, Italy
}

(Received 6 March 2013; accepted 28 June 2013; published online 22 July 2013)

We study the dynamics of the interface between two immiscible fluids in contact with a chemically homogeneous moving solid plate. We consider the generic case of two fluids with any viscosity ratio and of a plate moving in either directions (pulled or pushed in the bath). The problem is studied by a combination of two models, namely, an extension to finite viscosity ratio of the lubrication theory and a Lattice Boltzmann method. Both methods allow to resolve, in different ways, the viscous singularity at the triple contact between the two fluids and the wall. We find a good agreement between the two models particularly for small capillary numbers. When the solid plate moves fast enough, the entrainment of one fluid into the other one can occur. The extension of the lubrication model to the case of a non-zero air viscosity, as developed here, allows us to study the dependence of the critical capillary number for air entrainment on the other parameters in the problem (contact angle and viscosity ratio). @ 2013 AIP Publishing LLC. [http://dx.doi.org/10.1063/1.4814466]

\section{INTRODUCTION}

The problem of a solid plate pulled from a liquid bath has attracted considerable attention in the past including the seminal contributions from Landau, Levich, Derjaguin, and Bretherton. ${ }^{1-3}$ The problem has continued to be investigated, with a particular focus on the situation of partial wetting for which a dynamical wetting transition is observed: a liquid film is deposited only when a critical speed of withdrawal is exceeded (see Refs. 4 and 5 for reviews). Much less is known about the reversed case, where the solid is plunged into the liquid. Again, a dynamical wetting transition has been observed, now resulting in the entrainment of an air film or air bubbles. ${ }^{6-16}$ Despite the viscosity contrast between the liquid in the reservoir and that of the surrounding air, the dynamics inside the air is very important for this process. The perturbation analysis by $\operatorname{Cox}^{17}$ suggested that the critical speed is inversely proportional to the viscosity of the liquid, $1 / \eta_{\ell}$, with logarithmic corrections due to the viscosity of the air, $\eta_{g}$. This is similar to the scenario for air entrainment by viscous cusps, ${ }^{18,19}$ such as observed for impacting liquid jets..$^{20}$

Recently, in the experiment of plunging a plate into reservoirs of different liquids Marchand et al. ${ }^{8}$ observed that the dependence on $\eta_{\ell}$ is much weaker than predicted; enforcing a power-law fit to their data would give a small exponent, in between $-1 / 2$ and $-1 / 3$ rather than the expected -1 . This implies that air viscosity plays an important role on the onset of air entrainment even if it is orders of magnitude smaller than the liquid viscosity. The importance of air was already highlighted in similar dip-coating experiments, where a reduction of the ambient pressure was 
shown to significantly enhance the critical speed of entrainment. ${ }^{6}$ Yet, this raises another paradox: the dynamical viscosity of the air is virtually unchanged by a pressure reduction.

In this paper we provide a new framework to study air entrainment by advancing contact lines, in which the two-phase character of the flow is taken into account. The usual lubrication approximation is valid for small (liquid) contact angles and does not take into account the flow inside the gas. Since both assumptions are no longer valid near the onset of air entrainment, we extend the lubrication approximation such that it allows for large angles and a nonzero gas viscosity. This generalized lubrication theory is validated by comparing to Lattice Boltzmann simulations. We show that the meniscus shapes obtained from the generalized lubrication model agrees well with the simulations. Note, however, that such simulations are limited in terms of viscosity ratio and spatial resolution (i.e., the separation between the capillary length and the microscopic cutoff given by the interface width or, equivalently, by the effective slip length ${ }^{21}$ ), and cannot achieve experimental conditions for air entrainment. To compare to experiments and to explore the parameter space, in particular the importance of air viscosity $\eta_{g}$, we therefore provide a detailed study using the generalized lubrication model.

\section{FORMULATION}

We consider a smooth, chemically homogeneous solid plate translating across an interface of two immiscible fluids at a constant speed $U_{p}$ (positive/negative for plunging/withdrawing). As sketched in Fig. 1, the two fluids are contained in a reservoir much larger than all the lengths of the problem and the plate can be inclined to any angle $\alpha$. If the plate is not moving $\left(U_{p}=0\right)$ there is no flow in the fluids, and the interface equilibrates to a static shape due to balance between capillarity and gravity. The interface makes an equilibrium angle $\theta_{e}$ with the solid as a result of intermolecular interaction between the three phases at the contact line. Neglecting the contact angle hysteresis, $\theta_{e}$ takes a well-defined value determined by Young's law. The contact line equilibrates at a distance $\Delta$ above the bath, which can be expressed as

$$
\Delta= \pm \ell_{\gamma} \sqrt{2\left[1-\cos \left(\alpha-\theta_{e}\right)\right]} .
$$

Here, $\ell_{\gamma}=\sqrt{\frac{\gamma}{\left(\rho_{\ell}-\rho_{g}\right) g}}$ is the capillary length, defined by surface tension $\gamma$, gravity $g$, and the density difference $\rho_{\ell}-\rho_{g}$. The \pm sign depends on whether $\theta_{e}$ is smaller $( \pm)$ or larger ( - ) than the plate inclination $\alpha$. When addressing the transition to air entrainment, we will consider the upper phase A in Fig. 1 to be gaseous, while phase B is a liquid. We therefore use subscripts " $\ell$ " and " $g$ " to indicate liquid and gas phase, respectively.

When the plate is moving, the viscous drag generated by the moving plate gradually deforms the fluid-fluid interface. As long as the speed of the plate is lower than a threshold value, the meniscus equilibrates at a new distance $\Delta$ from the bath level (see Fig. 1). For positive $U_{p}$ the plate is moving downwards so that $\Delta$ is lower than the static equilibrium height, while the opposite holds for negative $U_{p}$. The contact line is assumed to be straight so that the problem can be treated as two-dimensional. The interface is described by the film thickness profile $h(x)$. The origin $x=0$ is chosen at the contact line. When the plate is moving beyond a critical speed, the meniscus can no longer equilibrate to a steady state. In the case of receding contact lines (withdrawing plate) this leads to the deposition of a liquid film, ${ }^{22-28}$ while air will be entrained for advancing contact lines. ${ }^{6-10,29-31}$

In this study we only focus on viscous flows, for which the Reynolds number is assumed to be zero. Thus for incompressible fluids, the flow fields in the fluids are described by Stokes equations and continuity,

$$
\eta_{g} \nabla^{2} \vec{u}_{g}-\vec{\nabla} p_{g}-\vec{\nabla} \Phi_{g}=0, \vec{\nabla} \cdot \vec{u}_{g}=0
$$

and

$$
\eta_{\ell} \nabla^{2} \vec{u}_{\ell}-\vec{\nabla} p_{\ell}-\vec{\nabla} \Phi_{\ell}=0, \vec{\nabla} \cdot \vec{u}_{\ell}=0,
$$

where $\vec{u}_{g}$ and $\vec{u}_{\ell}, p_{g}$ and $p_{\ell}, \Phi_{g}$ and $\Phi_{\ell}$ are the corresponding velocity fields, pressures and gravitational potentials in phase $\mathrm{A}$ and phase $\mathrm{B}$, respectively. When considering air entrainment, phase $\mathrm{A}$ 


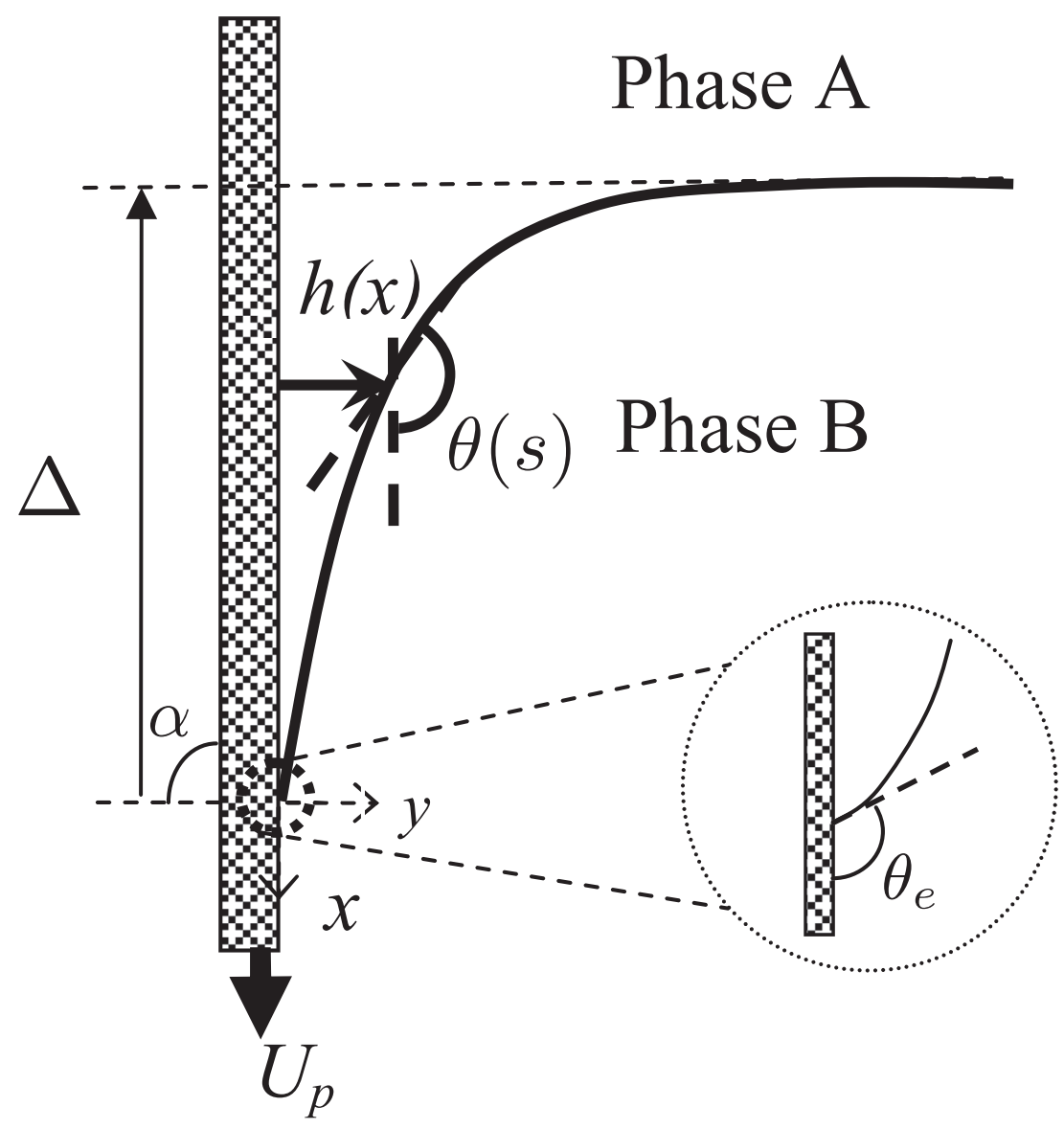

FIG. 1. Schematic diagram showing a plate moving with speed $U_{p}$ with respect to an interface of two immiscible fluids. The plate has an inclination $\alpha$, here drawn with $\alpha=\pi / 2$ for the case of a vertical plate. The interface touches the wall at the contact line with an angle assumed to be the same as the equilibrium contact angle $\theta_{e}$. The meniscus profile is described by $h(x)$ or the local angle $\theta(s)$, where $s$ is the arc length of the interface measured from the contact line. The total meniscus deformation is quantified by $\Delta$, the distance between the contact line and the level of the bath.

is assumed to be a gas of viscosity $\eta_{g}$, and phase B is a liquid of viscosity $\eta_{\ell}$. The relative viscosity is expressed by the viscosity ratio $R=\eta_{g} / \eta_{\ell}$ which, in practical situations, can be very small.

To solve for the flow fields and the interface shape, we need to specify appropriate boundary conditions. At the steady interface, the velocities parallel to the interface $u^{t}$ are continuous and the velocities normal to the interface $u^{n}$ vanish, so

$$
u_{g}^{t}=u_{\ell}^{t}
$$

and

$$
u_{g}^{n}=u_{\ell}^{n}=0 .
$$

In each phase, we define the normal stress $\sigma^{n} \equiv \hat{n} \cdot \widehat{\sigma} \cdot \hat{n}$ at the interface, where $\hat{n}$ is the unit vector normal to the interface and the stress tensor $\widehat{\sigma}$ is defined as (in cartesian coordinates)

$$
\sigma_{i j} \equiv-p \delta_{i j}+\eta\left(\frac{\partial u_{i}}{\partial x_{j}}+\frac{\partial u_{j}}{\partial x_{i}}\right)
$$

The normal stress discontinuity across the interface is related to the curvature $\kappa$ and to the surface tension $\gamma$ by Laplace's law

$$
\sigma_{\ell}^{n}-\sigma_{g}^{n}=\gamma \kappa
$$


By contrast, the tangential stress component $\sigma^{t} \equiv \hat{t} \cdot \widehat{\sigma} \cdot \hat{n}$ is continuous across the interface,

$$
\sigma_{g}^{t}=\sigma_{\ell}^{t}
$$

At the solid/fluid boundary $(y=0)$, the velocity normal to the wall $u_{y}(y=0)$ vanishes as no penetration of fluid through the solid is allowed, i.e.,

$$
u_{y}(y=0)=0 .
$$

Regarding the velocity component parallel to the wall, $u_{x}(y=0)$, the situation is more subtle because of the moving contact line singularity: ${ }^{4,32}$ imposing a no-slip boundary condition leads to diverging stress fields and calls for a microscopic mechanism of regularization. In Sec. III we present two methods to solve the flow equations, which naturally involve different regularizations of the singularity: a generalized lubrication model and Lattice Boltzmann simulations. The microscopic boundary condition will therefore be discussed separately below.

\section{METHODS}

In this section we present two methods to determine the meniscus shape sketched in Fig. 1. We first present a model that can be considered as a generalization of the standard lubrication approximation. We then present the Lattice Boltzmann method, which is a rather different approach to solve for the flow and the meniscus shape. The models will be refereed to as GL (Generalized Lubrication model) and LB (Lattice Boltzmann).

\section{A. Generalized lubrication model}

\section{Derivation}

The lubrication approximation has been a very efficient framework to deal with thin film flows. ${ }^{33}$ This systematic reduction of the Navier-Stokes equations is very suitable for numerical simulations and in many cases allows for analytical results. ${ }^{4}$ It is usually derived for flows that involve a single phase that constitutes a "thin" film, i.e., the slopes $d h / d x$ are assumed small. However, the expansion parameter underlying the analysis is not the interface slope, but the capillary number $\mathrm{Ca}=U \eta_{\ell} / \gamma .{ }^{33}$ This means that a lubrication-type analysis can be performed whenever surface tension dominates over viscosity. Indeed, it was shown in Ref. 34 that the lubrication approximation can be generalized to large interface angles $\theta$, giving a perfect agreement with the perturbation results by Voinov ${ }^{35}$ and Cox. ${ }^{17}$ Here we further extend this approach by taking into account, besides of the effect of a large slope, also of the viscous flows on both sides of the interface. The goal is to provide a model that can deal with moving contact lines in cases where both phases are important (as in Fig. 1). In particular, this will allow us to study the air entrainment transition. Let us now derive this generalized lubrication model. As mentioned in Sec. II, the interface curvature $\kappa$ is determined by the normal stress difference across the interface. In curvilinear coordinates, we write

$$
\gamma \kappa \equiv \gamma \frac{d \theta}{d s}=\sigma_{\ell}^{n}-\sigma_{g}^{n}
$$

where $\theta$ is the local interface angle and $s$ is the arc length (see Fig. 1). The normal stresses have to be determined from the flows in the fluids, which themselves depend on the shape of the interface. For the usual lubrication theory in which the interface slope is small, the leading order contribution to the flow reduces to a parabolic Poiseuille flow inside the film. This can be generalized to two-phase flows and large interface slopes: as long as the capillary number is small, the interface curvature is small as well and the leading order velocity field is given by the flow in a wedge (Fig. 2). The wedge solutions have been obtained analytically by Huh and Scriven, ${ }^{32}$ for any viscosity ratio $R=\eta_{g} / \eta_{\ell}$ and for any wedge angle $\theta$. Figure 2 shows the corresponding streamlines. Based on these exact solutions, the local normal stress can be determined, thus giving the local curvature of the interface through Eq. (10). 


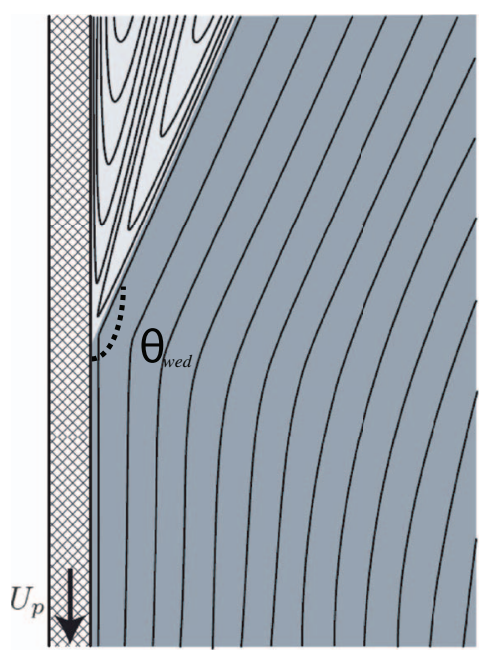

FIG. 2. Streamlines of the flow in a wedge of angle $\theta_{\text {wed }}$, which in this case is close to $\pi$. This flow solution has been derived analytically by Huh and Scriven, ${ }^{32}$ and is used here to derive a generalized lubrication model.

We denote the quantities derived from the Huh-Scriven solutions by capital symbols, e.g., normal stress is denoted by $\Sigma^{n}$, velocity by $\vec{U}$, and pressure by $P$. For the Huh-Scriven solutions, it turns out that the non-isotropic part of the normal stress at the interface vanishes so that

$$
\Sigma^{n}=-P .
$$

Approximating the normal stresses by the Huh-Scriven solutions, Eq. (10) becomes

$$
\gamma \frac{d \theta}{d s}=\Sigma_{\ell}^{n}-\Sigma_{g}^{n}=P_{g}-P_{\ell}
$$

Since $P_{g}-P_{\ell}$ are defined up to an integration constant, it is convenient to differentiate Eq. (12) once with respect to $s$, giving

$$
\gamma \frac{d^{2} \theta}{d s^{2}}=\frac{d P_{g}}{d s}-\frac{d P_{\ell}}{d s}=\left[\vec{\nabla} P_{g}-\vec{\nabla} P_{\ell}\right]_{\mathrm{int}} \cdot \hat{e}_{s} .
$$

The index "int" indicates that the quantities inside the brackets are to be evaluated on the interface and $\hat{e}_{s}=\hat{t}$ is the unit vector tangent to the interface.

When Stokes equation (2) is applied, Eq. (13) can be rephrased in terms of Huh-Scriven velocity fields

$$
\gamma \frac{d^{2} \theta}{d s^{2}}=\left[\eta_{g} \nabla^{2} \vec{U}_{g}-\eta_{\ell} \nabla^{2} \vec{U}_{\ell}-\vec{\nabla}\left(\Phi_{g}-\Phi_{\ell}\right)\right]_{\mathrm{int}} \cdot \hat{e}_{s} .
$$

The viscous contributions on the right-hand side can be expressed in terms of $R$ and $\theta$ in the form

$$
\eta_{\ell}\left[\frac{\eta_{g}}{\eta_{\ell}} \nabla^{2} \vec{U}_{g}-\nabla^{2} \vec{U}_{\ell}\right]_{i n t} \cdot \hat{e}_{s}=\frac{3 \eta_{\ell} U_{p} f(\theta, R)}{h^{2}},
$$

where

$$
\begin{aligned}
f(\theta, R) & \equiv \frac{2 \sin ^{3} \theta\left[R^{2} f_{1}(\theta)+2 R f_{3}(\theta)+f_{1}(\pi-\theta)\right]}{3\left[R f_{1}(\theta) f_{2}(\pi-\theta)-f_{1}(\pi-\theta) f_{2}(\theta)\right]}, \\
f_{1}(\theta) & \equiv \theta^{2}-\sin ^{2} \theta, \\
f_{2}(\theta) & \equiv \theta-\sin \theta \cos \theta, \\
f_{3}(\theta) & \equiv\left(\theta(\pi-\theta)+\sin ^{2} \theta\right) .
\end{aligned}
$$

The gravity terms in (14) can be simplified to

$$
\vec{\nabla}\left[\Phi_{g}-\Phi_{\ell}\right]_{i n t} \cdot \hat{e}_{s}=-\left(\rho_{\ell}-\rho_{g}\right) g \sin (\theta-\alpha),
$$

where $\rho_{g}$ and $\rho_{\ell}$ are the densities of the two phases. 
The final result of the analysis is a generalized form of the lubrication equation, which after scaling all lengths with the capillary length $l_{\gamma}=\sqrt{\frac{\gamma}{\left(\rho_{\ell}-\rho_{g}\right) g}}$ becomes

$$
\frac{d^{2} \theta}{d s^{2}}=\frac{3 \mathrm{Ca}}{h^{2}} f(\theta, R)+\sin (\theta-\alpha)
$$

In the reminder we will use the same symbols for rescaled lengths. Once more, the capillary number is defined based on the viscosity of the liquid, $\mathrm{Ca}=\eta_{\ell} U_{p} / \gamma$. This equation must be complemented by the geometrical relation

$$
\frac{d h}{d s}=\sin \theta .
$$

Note that for all numerical examples in the rest of the paper, we consider a vertical plate, for which $\alpha=\pi / 2$.

One easily verifies that the standard lubrication equation is recovered when taking the limit of vanishing $R, \theta$, and $\alpha$. Namely, $f(0,0)=-1$ and (18) reduces to

$$
h^{\prime \prime \prime}=\frac{3 \mathrm{Ca}}{h^{2}}-h^{\prime}+\alpha .
$$

When considering a single phase with arbitrary angle, one recovers the equation previously proposed in Ref. 34, with $f(\theta, 0)$ instead of $f(\theta, R)$.

\section{Slip boundary condition}

It is important to note that Eq. (18) is derived from the Huh-Scriven solution with no-slip boundary condition. Near the contact line, however, this induces a divergence of the pressure and of the shear stress, which scale as $\sim \eta_{\ell} U_{p} / h$ and $\sim \eta_{g} U_{p} / h$ in the liquid and the gas, respectively. A purely hydrodynamic approach to regularize the singularity is to impose a slip boundary condition on the solid wall. No analytical solution for the flow in a wedge with slip can be obtained. However, as contact line flows are only mildly affected by the details of the microscopic conditions, ${ }^{4,5,36}$ as for example, can be seen in the Cox-Voinov law for one-phase case contact line motion, the dependence on the microscopic cutoff length (e.g., slip length) is of a weak logarithmic, here we proceed by a phenomenological regularization. We therefore consider the standard lubrication equation, which can be derived including a Navier slip boundary condition. It reads

$$
h^{\prime \prime \prime}=\frac{3 \mathrm{Ca}}{h\left(h+3 \lambda_{s}\right)}-h^{\prime}+\alpha,
$$

where $\lambda_{s}$ is the slip length. In comparison to (20), the effect of slip can be summarized by a correction factor $h /\left(h+3 \lambda_{s}\right)$ for the viscous term. Indeed, this weakens the singularity such that the equations can be integrated to $h=0 .{ }^{37}$ We simply propose to use the same regularization factor for the viscous term in (18), i.e.,

$$
\frac{d^{2} \theta}{d s^{2}}=\frac{3 \mathrm{Ca}}{h\left(h+3 \lambda_{s}\right)} f(\theta, R)+\sin (\theta-\alpha),
$$

where we have assumed the slip length to be independent of $R$. The appropriate boundary conditions are that the equilibrium contact angle $\theta_{e}$ is recovered at the contact line, and that the interface attains the angle of the reservoir at infinity:

$$
h(s=0)=0 ; \theta(s=0)=\theta_{e} ; \theta(s \rightarrow \infty)=\alpha .
$$

The meniscus shape is now fully determined by the lubrication equation (22), geometry (19) and boundary conditions (23). For a given value of the capillary number $\mathrm{Ca}$, the model parameters are the viscosity ratio $R$, the contact angle $\theta_{e}$, and the microscopic length $\lambda_{s}$. Below, we compute the shape of the meniscus for different parameters by numerical integration, using a 4th order Runge-Kutta numerical scheme. As the boundary conditions are imposed at different locations, the solution is determined using a shooting algorithm. 


\section{B. Lattice Boltzmann method}

In this section we discuss some of the key features of the LB method. In a LB model, the following discrete Boltzmann equation is solved for the single particle distribution function $f_{i, \alpha}(\mathbf{x}, t)$ over a $2 \mathrm{D}$ square lattice:

$$
f_{i, \alpha}\left(\mathbf{x}+\mathbf{e}_{i} \Delta t, t+\Delta t\right)-f_{i, \alpha}(\mathbf{x}, t)=\Omega_{i, \alpha}(\mathbf{x}, t),
$$

where

$$
\Omega_{i, \alpha}(\mathbf{x}, t)=-\frac{1}{\sigma_{\alpha}}\left[f_{i, \alpha}(\mathbf{x}, t)-f_{i, \alpha}^{\mathrm{eq}}(\mathbf{x}, t)\right]
$$

is the single time relaxation, linear BGK collision operator, ${ }^{38} f_{i, \alpha}^{\mathrm{eq}}(\mathbf{x}, t)$ is the discrete Maxwell distribution function defined as

$$
f_{i, \alpha}^{\mathrm{eq}}(\mathbf{x}, t)=\rho_{\alpha} w_{i}\left[1+\frac{\mathbf{e}_{i} \cdot \mathbf{u}_{\alpha}}{c_{s}^{2}}+\frac{\mathbf{e}_{i} \cdot \mathbf{u}_{\alpha}}{2 c_{s}^{4}}-\frac{\mathbf{u}_{\alpha} \cdot \mathbf{u}_{\alpha}}{2 c_{s}^{2}}\right],
$$

where

$$
\rho_{\alpha}=\sum_{i} f_{i, \alpha}, \quad \rho_{\alpha} \mathbf{u}_{\alpha}=\sum_{i} f_{i, \alpha} \mathbf{e}_{i},
$$

and $w_{i}, \mathbf{e}_{i}$ in (25) are the weights and the corresponding lattice speeds, respectively. ${ }^{39,40}$ The weights, $w_{i}$, corresponding to the two-dimensional and 9 velocity model, D2Q9, are given by $w_{0}=4 / 9, w_{1}=w_{2}=w_{3}=w_{4}=1 / 9$, and $w_{5}=w_{6}=w_{7}=w_{8}=1 / 36$. The total fluid density is $\rho=\sum_{\alpha} \rho_{\alpha}$ and the total hydrodynamic velocity is $\mathbf{u}=\sum_{\alpha} \rho_{\alpha} \mathbf{u}_{\alpha} / \rho$. The effective kinematic viscosity is related to the relaxation time of the different components $v=\sum_{\alpha} c_{s}^{2}\left(\sigma_{\alpha} c_{\alpha}-0.5\right),{ }^{41}$ $c_{\alpha}=\rho_{\alpha} / \rho$ is the concentration, and $c_{s}=1 / \sqrt{3}$ is the speed of sound on the lattice. In absence of an external force, each component satisfies the ideal gas equation of state $p=c_{s}^{2} \rho$. For multicomponent simulation we are using two distribution functions $(\alpha=1,2)$, whereas for multiphase simulations we restrict ourselves to only one distribution function $(\alpha=1)$.

\section{Multiphase/multicomponent model}

The multicomponent/multiphase algorithm is based on a standard Shan-Chen lattice Boltzmann method. ${ }^{41-43}$ The non-ideal nature of the fluid is introduced by adding an internal force and shifting the lattice Boltzmann equilibrium velocity as

$$
\mathbf{u}_{\alpha}^{\mathrm{eq}}=\mathbf{u}^{\prime}+\frac{\sigma_{\alpha} \mathbf{F}^{\alpha}}{\rho_{\alpha}}, \quad \text { where } \quad \mathbf{u}^{\prime}=\frac{\sum_{\alpha} \rho_{\alpha} \mathbf{u}_{\alpha} / \sigma_{\alpha}}{\sum_{\alpha} \rho_{\alpha} / \sigma_{\alpha}} .
$$

For the non-ideal interaction the force $\mathbf{F}_{\alpha}$ in the Shan-Chen model ${ }^{41,43}$ is given by

$$
\mathbf{F}_{\alpha}=-G_{\alpha \alpha^{\prime}} \psi_{\alpha}(\mathbf{x}) \sum_{i, \alpha \neq \alpha^{\prime}} w_{i} \psi_{\alpha^{\prime}}\left(\mathbf{x}+\mathbf{e}_{i}\right) \mathbf{e}_{i},
$$

where $\left\{\alpha, \alpha^{\prime}\right\}=\{1,2\}$ are indices for two fluid components while the coupling parameter $G_{\alpha \alpha^{\prime}}$ is the strength of the interaction and determines the surface tension in the model. This force allows for the spontaneous formation of an interface between the different fluid components, i.e., no interface tracking is needed. For multicomponent simulations $G_{12}=G_{21}=G, G_{11}=G_{22}=0$, $\psi_{\alpha}=\rho_{\alpha}$. In the case of multiphase simulations $\alpha=1, \psi=1-\exp (-\rho)$. The equation of state is modified to $p=c_{s}^{2}\left(\rho_{1}+\rho_{2}\right)+G c_{s}^{2} \rho_{1} \rho_{2}$ and $p=c_{s}^{2} \rho+\frac{G}{2} c_{s}^{2} \psi^{2}$, respectively, for multicomponent and multiphase simulations, where the first term corresponds to the ideal gas and the second term is the non-ideal part due to the external Shan-Chen force. Many validation studies exist, showing that the hydrodynamical fields, $\mathbf{u}(\mathbf{x}, t), \rho(\mathbf{x}, t)$ satisfies the Navier-Stokes equations with a non-ideal Pressure tensor, under a suitable multiscale Chapman-Enskog expansion. 


\section{Boundary conditions for LB simulations}

The no-slip boundary condition for the fluid corresponds to the bounce back boundary condition ${ }^{39}$ for the distribution functions $f_{i, \alpha}(\mathbf{x}, t)$ defined at the boundary nodes.

The surface wetting for multicomponent simulations is introduced by adding an additional force at the wall ${ }^{44,45}$

$$
\mathbf{F}_{\alpha}^{a d s}=-G_{\alpha}^{a d s} \rho_{\alpha}(\mathbf{x}, t) \sum_{i} w_{i} s\left(\mathbf{x}+\mathbf{e}_{i}\right) \mathbf{e}_{i},
$$

where $s\left(\mathbf{x}+\mathbf{e}_{i}\right)=1$ for a wall node and is 0 for a fluid node. The parameter $G_{\alpha}^{a d s}$ can be varied to control the wetting properties of the wall; in all our simulations we have used $G_{1}^{a d s}=-G_{2}^{a d s}$. Similarly for multiphase simulations we fix a wetting parameter, $\rho_{w}$ for the nodes in the wall and calculate the Shan-Chen force (28) at the wall ${ }^{44}$

$$
\mathbf{F}^{a d s}=-G \psi\left(\rho_{w}\right) \sum_{i} w_{i} s\left(\mathbf{x}+\mathbf{e}_{i}\right) \mathbf{e}_{i},
$$

where $s\left(\mathbf{x}+\mathbf{e}_{i}\right)=1$ for a wall node and is 0 for a fluid node. The parameter $\rho_{w}$ is varied to control the equilibrium contact angle at the wall. Let us stress that all LB methods, independent of the underlying kinetic model, describe the multi-phase or the multi-component dynamics via a diffuse interface approach. There exists therefore a natural regularizing microscopic length which is on the order of the interface width, typically a few grid points. Such a length scale is also on the order of the effective slip length that must be used whenever a quantitative comparison between the hydrodynamical behavior of the LB and the evolution of the equivalent Navier-Stokes system is made, as shown recently, for example, in Ref. 21.

\section{COMPARING THE LUBRICATION MODEL AND LATTICE BOLTZMANN SIMULATIONS}

In this section we compare the results of the GL model and the LB simulations. Since the latter are limited to moderate viscosity ratios $R$, the comparison is done for $R=0.03,0.8$, and 1 . We further explore the parameter space in Sec. V, using only the lubrication approach.

The results for this section are computed for $\theta_{e}=\pi / 2$ and $\alpha=\pi / 2$. The lattice separation in LB is 0.01 (in capillary length units), which will be related to an effective slip length from the comparison with the lubrication model. We will see that this lattice separation corresponds to effective slip length of 0.002 , which is larger than the slip length of common fluids $\left(\lambda_{s}=10^{-6}-10^{-5}\right)$. However, in order to obtain results for realistic values of effective slip length, we need to do simulations with larger capillary length (characteristic length scale). This leads to computationally expensive for lattice Boltzmann simulations. For instance, reducing slip length by one order of magnitude will require 10 times bigger LBM simulation domain size and time. This is why we choose a moderate value of slip length $=0.002$ for our comparisons.

\section{A. Meniscus rise}

We first compare the meniscus rise $\Delta$ for viscosity ratios $R=0.03,0.8$, and 1 in Fig. 3. When the plate is at rest, $\mathrm{Ca}=0$, the meniscus is perfectly horizontal $\Delta=0$ due to the choice of $\theta_{e}$ and $\alpha$. Let us first consider the case where both liquids have identical viscosity, $R=1$, but are still immiscible due to the nonzero surface tension. This case is perfectly symmetric in the sense that $\mathrm{Ca} \rightarrow-\mathrm{Ca}$ gives $\Delta \rightarrow-\Delta$ : there is no difference between plunging and withdrawing. This symmetry is indeed observed in Fig. 3. Blue diamonds represent LB simulations, while the dashed-dotted line is the GL model. We use this symmetric case to calibrate the microscopic parameter of the GL model. A nearly perfect fit is achieved for slip length $\lambda_{s}=0.002$, which is a reasonable value given that the grid size used in the LB simulation is 0.01 . As Ca increases, the viscous forces increasingly deform the interface, leading to a change in $\Delta$.

It is interesting to see to what extent the same microscopic parameter $\lambda_{s}$ is able to describe different viscosity ratios. We first mildly decrease the viscosity ratio to $R=0.83$ and still find a 


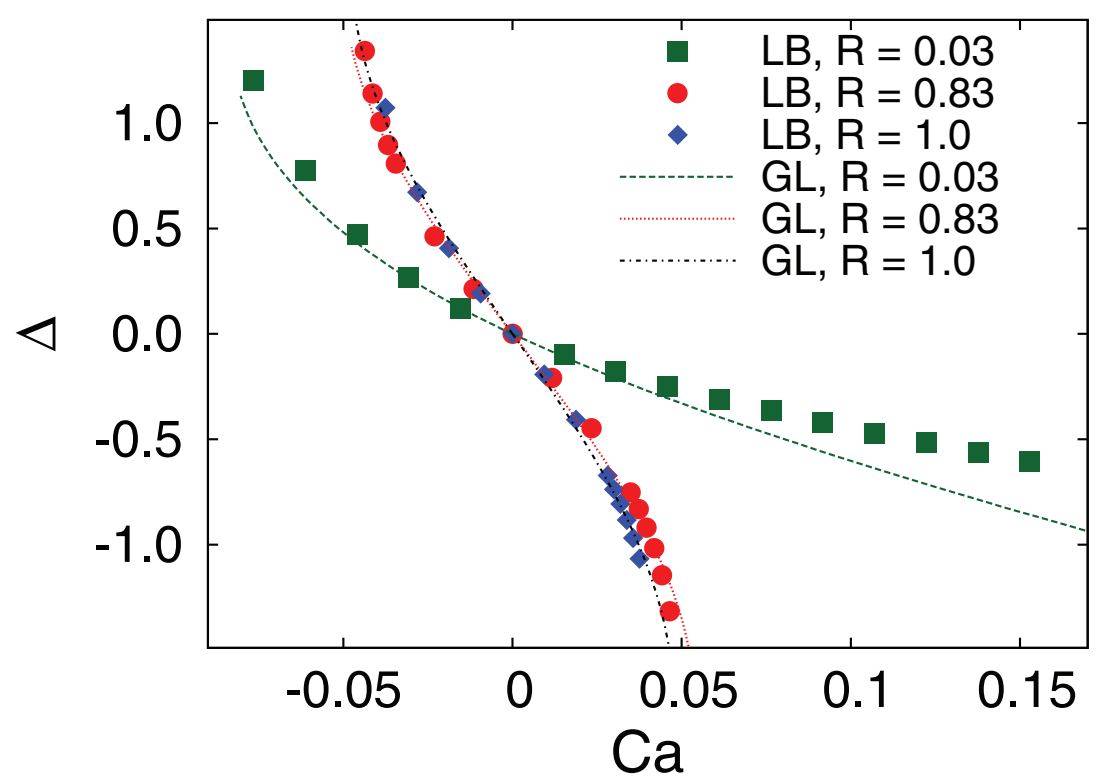

FIG. 3. Meniscus deformation $\Delta$ as a function of $\mathrm{Ca}$ for $\theta_{e}=\pi / 2$. Ca is negative/positive when the plate is moving upward/downward. Symbols: Results of Lattice Boltzmann (LB) simulation. Lattice separation $=0.01$. Curves: Results of the lubrication-type (GL) model with a slip length $\lambda_{s}=0.002$. All lengths are scaled by the capillary length $\ell_{\gamma}$.

very good agreement between LB an GL (red circles and dotted line, respectively). With respect to the case $R=1$ we see that $|\Delta|$ is slightly smaller at a given value of Ca. This means that for the same speed, the meniscus is deformed by a smaller amount due to the lower viscosity of the upper phase. When further decreasing the viscosity ratio to $R=0.03$ (green squares, dashed line), some differences between LB and GL becomes apparent (the same value for $\lambda_{s}$ is maintained). The meniscus in GL is systematically below the value obtained in LB. A possible explanation for this difference is the sensitivity of the result on the microscopic contact angle imposed as a boundary condition. Still, both models agree reasonably well and display very similar trends. In particular, we find that much larger values of $\mathrm{Ca}$ can be achieved due to the strong reduction of the viscosity in the upper phase. This effect is most pronounced for the plunging case, for which the liquid is advancing. This is consistent with experimental observations that advancing contact lines can move much more rapidly than receding contact lines. ${ }^{4,5,8}$ The breakdown of the steady solutions, which signals the transition to air/liquid entrainment, will be discussed in details in Sec. V.

\section{B. Shape of the meniscus}

A much more detailed test for the two models is to investigate the detailed structure of the interface: How well do the shapes of the menisci compare between GL and LB? In Fig. 4, we show the dynamical meniscus profiles for $\mathrm{Ca}=0.019,0.028$, and 0.036 , in the case of equal viscosities, $R=1$. Note that the contact line position is held constant at $x=0$, so that the bath appears at different heights due to the increase in magnitude of $\Delta$ with speed. The agreement of the results of GL model and LB simulation is very good in particular for $\mathrm{Ca}=0.019$ and 0.028 , even down to the contact line region (Fig. 4(b)). For larger plate velocities some differences become apparent. These differences may be due to different reasons. First, one has to notice that a large $\mathrm{Ca}$ is also accompanied by a large viscous stress contribution, leading to a larger bending of the interface and therefore to the possibility to leave the realm of application of the GL model. Second, as said, in the LB approach the effective slip length is an output and not an input as for the GL, and it is not clear that one would need a finer tuning of it as a function of the capillary number, in order to match the GL behavior. 

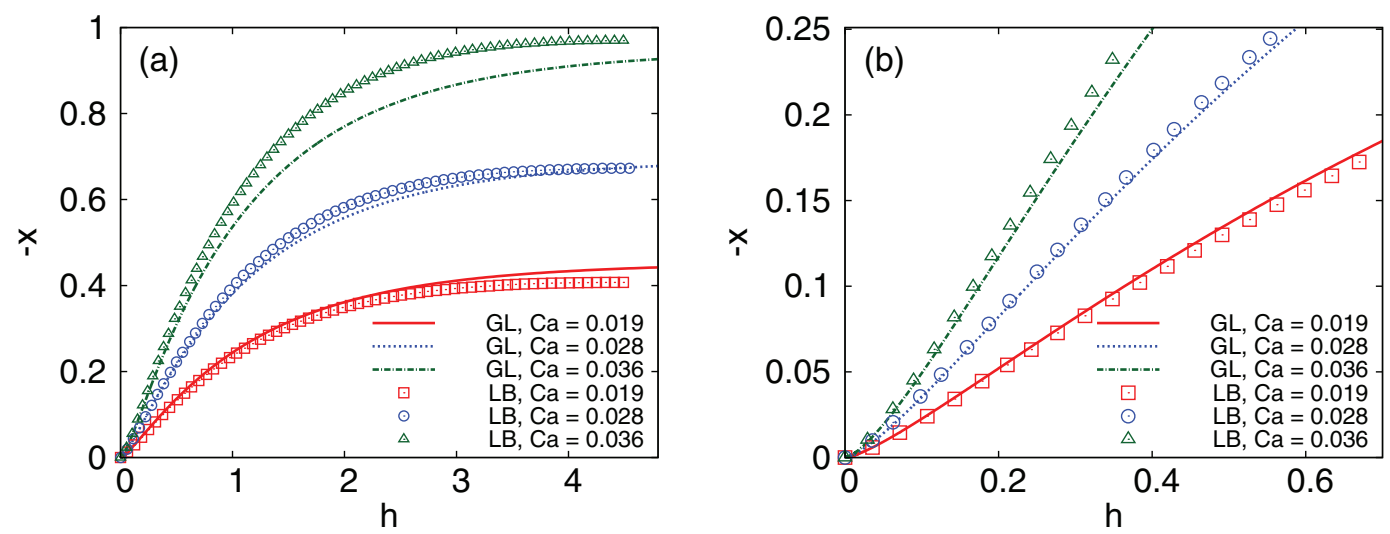

FIG. 4. Dynamical meniscus profiles $-x$ vs $h$ for $R=1\left(\theta_{e}=\pi / 2\right.$ and $\lambda_{s}=0.002$ for the GL model, lattice separation $=0.01$ for the LB simulation). All lengths have been scaled by the capillary length. The contact line is at $x=0$ so that the bath is at different $x$ for different Ca. (a) Curves: Results of GL model. Symbols: Results of LB simulation. (b) Zoom in on the contact line region.

An even more detailed characterization of the meniscus shape is provided by the local angle of the interface $\theta$ vs $h$, see Fig. 5. Clearly, both the GL model and the LB simulation exhibit the same nontrivial variation of the contact angle. At small scales, the angle approaches $\theta_{e}=\pi / 2$, while at large scale the meniscus evolves towards the reservoir $\theta=\alpha=\pi / 2$. In between, the angle changes significantly due to the well-known effect of "viscous bending":4 the balance of viscosity and surface tension leads to a curvature of the interface. Very similar variations of the meniscus angle have been obtained experimentally. ${ }^{46}$

\section{MAXIMUM SPEEDS AND TRANSITION TO AIR ENTRAINMENT}

In this section we discuss the physics of air entrainment in the case of a plunging plate $(\mathrm{Ca}>0)$. For realistic situations the viscosity ratio $R$ is typically very small, of order $10^{-2}$ for water and much smaller for very viscous liquids. This regime can be accessed by the GL model only, as LB is restricted to moderate viscosity contrasts. In the first part of this section we discuss how the transition to air entrainment is captured in our model in terms of a bifurcation diagram. Next we study the effect of viscosity ratio on the critical speed. In the last part we investigate how the critical speed depends on the microscopic parameters such as the slip length and the static contact angle.
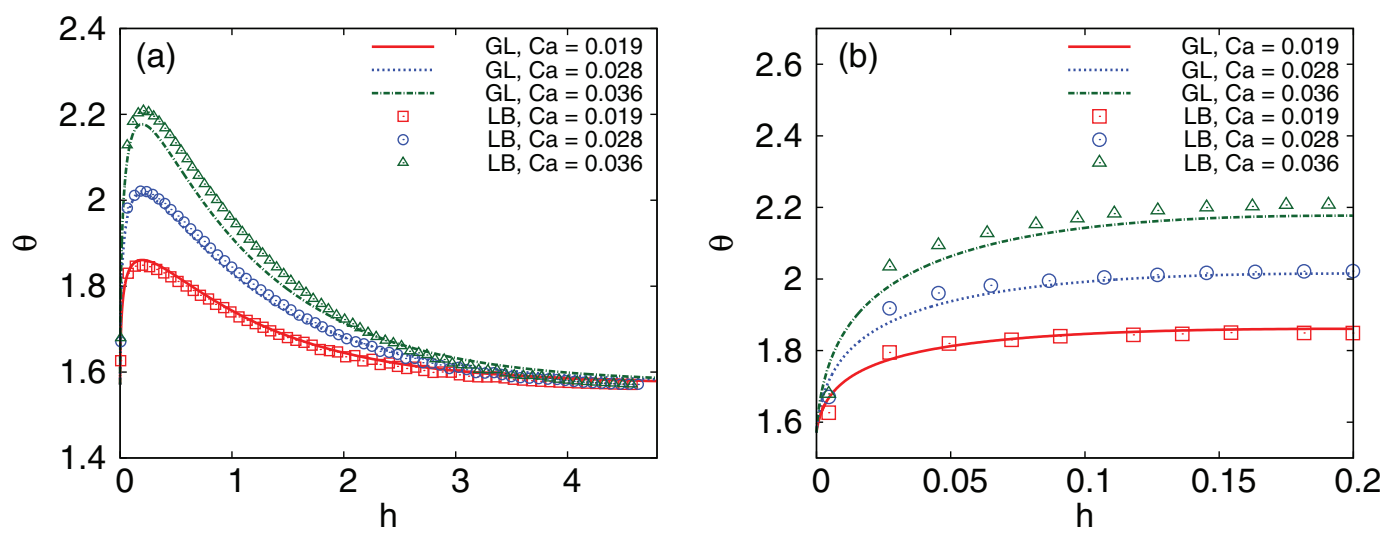

FIG. 5. Local meniscus angle $\theta$ vs $h$ for $R=1$. Identical parameters as in Fig. 4. 


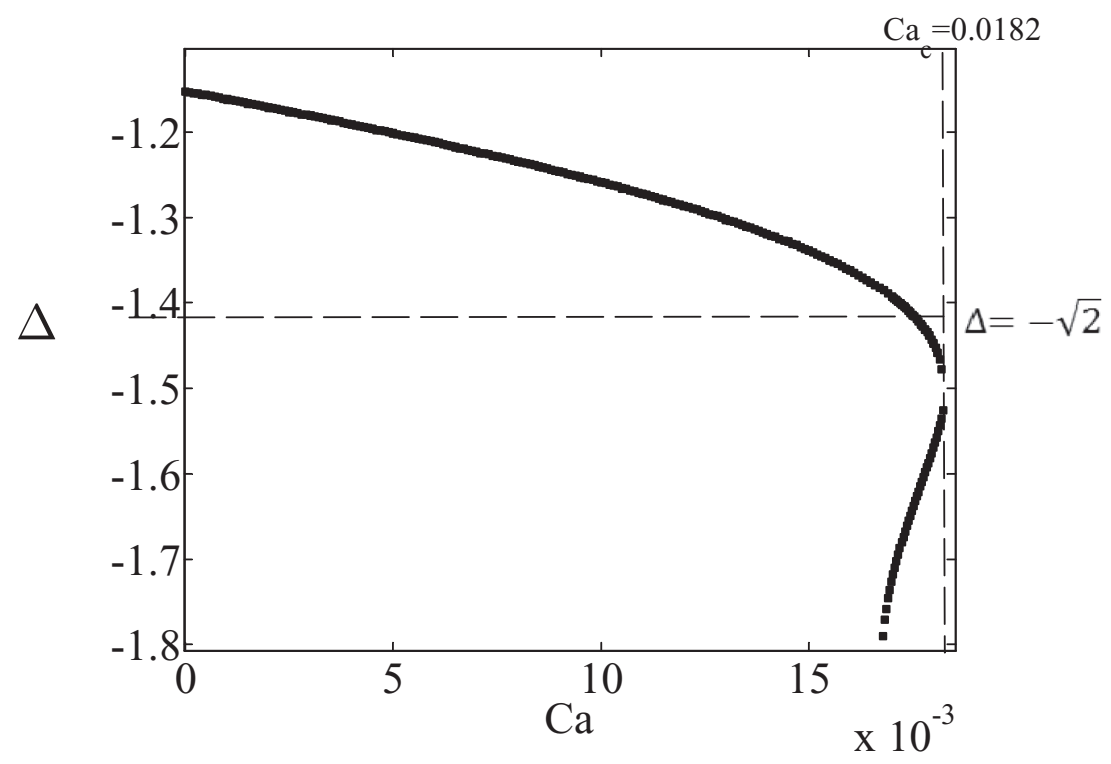

FIG. 6. Meniscus fall $\Delta$ versus $\mathrm{Ca}\left(\theta_{e}=2.8 \mathrm{rad}, R=0.01, \lambda_{s}=10^{-5}\right)$. The horizontal dashed line indicates the minimum value of $\Delta$ for a static meniscus $\left(=-\sqrt{2}\right.$, with $\left.\theta_{e}=\pi\right)$.

\section{A. Maximum speed for advancing contact lines}

We first consider a case where the equilibrium contact angle is close to $\pi$. For such a hydrophobic substrate we expect air entrainment to occur at relatively small $\mathrm{Ca},{ }^{9,10}$ and therefore relatively weak curvature of the interface. This is important, since the assumption underlying the GL model is that the interface curvature is weak. ${ }^{34}$ We will therefore focus on $\theta_{e}=2.8$ rad and explicitly verify how strongly the interface is curved for our numerical solutions. For most fluids, the slip length is in order of magnitude of $1-10 \mathrm{~nm}$ which corresponds to $\lambda_{s}=10^{-6}-10^{-5}$. Thus in the following calculations, we take $\lambda_{s}=10^{-5}$. Figure 6 shows the drop of the meniscus $\Delta$ as function of Ca for a viscosity ratio $R=0.01\left(\lambda_{s}=10^{-5}\right.$, i.e., on the order of $\left.10 \mathrm{~nm}\right)$. As Ca increases, the contact line equilibrates at a lower position resulting in a more negative value of $\Delta$. However, when Ca achieves a certain critical value, stationary solutions cease to exist. This corresponds to a maximum plate velocity, or critical capillary number $\mathrm{Ca}_{\mathrm{c}}$. By analogy to deposition of liquid films for plate withdrawal, ${ }^{26-28,47,48}$ this can be identified as the onset of air entrainment: above $\mathrm{Ca}_{c}$, unsteady solutions will develop, with a downward motion of the contact line. ${ }^{8}$ As can be seen in Fig. 6, the critical point arises close to $\Delta=-\sqrt{2}$, which according to (1) corresponds to a meniscus with apparent contact angle $\pi$. This is the analogue of the withdrawal case, for which $\Delta=+\sqrt{2}$ and the apparent contact angle vanishes at the transition. ${ }^{23,27}$ Note that viscous effects push system slightly below this maximum extent of deformation for a perfectly static meniscus, with the critical point slightly below $\Delta=-\sqrt{2}$.

Interestingly, for a range of speeds $\mathrm{Ca}<\mathrm{Ca}_{\mathrm{c}}$ one actually finds more than one solution (cf. Fig. 6). Upon decreasing $\Delta$, the capillary number first increases and then decreases close to the critical point. We refer to the solution branches around $\mathrm{Ca}_{c}$ as the upper and lower branch, respectively. Once again, an identical bifurcation structure was previously observed for the withdrawing plate case. ${ }^{27,49,50}$ To compare these two types of solutions, we show the corresponding meniscus profiles for $\mathrm{Ca}=0.017$ in Fig. 7. At a large distance from the contact line, the solutions are almost identical in shape. Zooming in on the contact line region, however, we see the lower branch (red dashed curve) solution displays a "finger" that explains the larger magnitude of $\Delta$ with respect to the upper branch.

\section{B. Effect of viscosity ratio}

A key parameter for the transition to air entrainment is the viscosity ratio $R$. Figure 8 shows the meniscus fall $\Delta$ as function of Ca for different viscosity ratios: $R=0,10^{-4}, 10^{-3}$, and $10^{-2}$. 


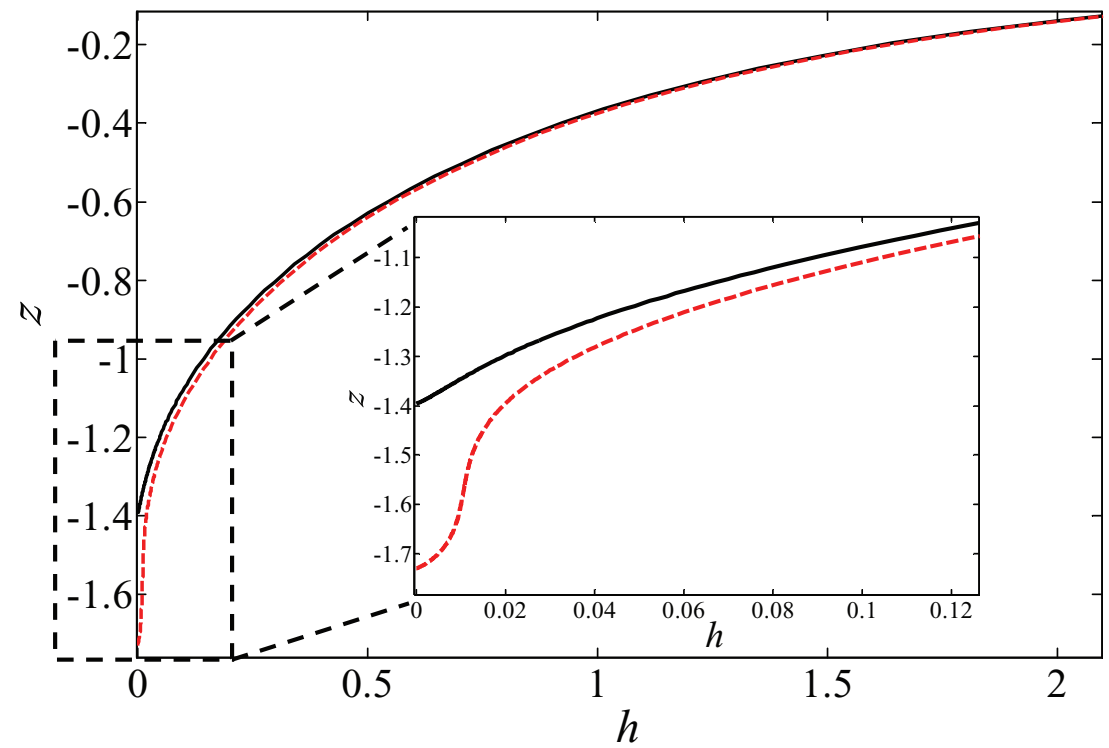

FIG. 7. Meniscus profiles for the upper branch (black solid curve) and the lower branch (red dashed curve) solutions for $\mathrm{Ca}=0.017\left(\theta_{e}=2.8 \mathrm{rad} ., R=0.01, \lambda_{s}=10^{-5}\right)$. Here we define $z=\Delta-x$. So the bath level is at $z=0$.

At $\mathrm{Ca}=0$, all cases have the same value of $\Delta=-1.15$ corresponding to a static bath with contact angle $\theta_{e}=2.8$. Now we consider the result for $R=0$, for which there are no viscous effects in the air $\left(\eta_{g}=0\right)$. We observe that $\Delta$ decreases with $\mathrm{Ca}$, but without any bifurcation. It appears that steady meniscus solutions can be sustained up to arbitrarily large plate velocities. In fact, the curve is consistent with the scaling $\Delta \sim-\sqrt{\mathrm{Ca}}$ at relatively large $\mathrm{Ca}$, corresponding to a simple balance between gravity and viscosity. For $R \neq 0$, however, the situation becomes fundamentally different. While the curves follow the same trend as for $R=0$ at small $\mathrm{Ca}$, a deviation appears at larger speeds that ultimately leads to a critical point. Each nonzero viscosity ratio has a well-defined critical speed, with $\mathrm{Ca}_{c}$ increasing when the viscosity ratio $R$ is reduced.

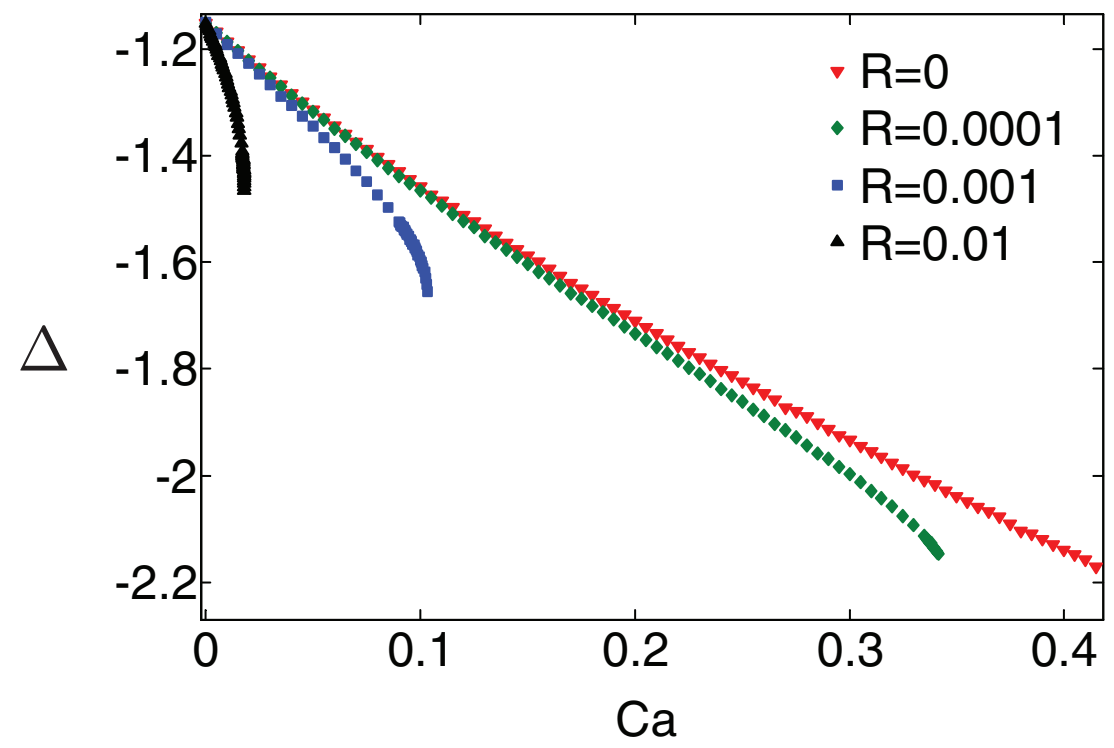

FIG. 8. Meniscus fall $\Delta$ versus Ca for different viscosity ratios $\left(\theta_{e}=2.8 \mathrm{rad}, \lambda_{s}=10^{-5}\right)$. For the case the gas phase has no viscosity, $R=0$, steady-state menisci can be maintained to arbitrarily large velocity (within our numerical resolution). 


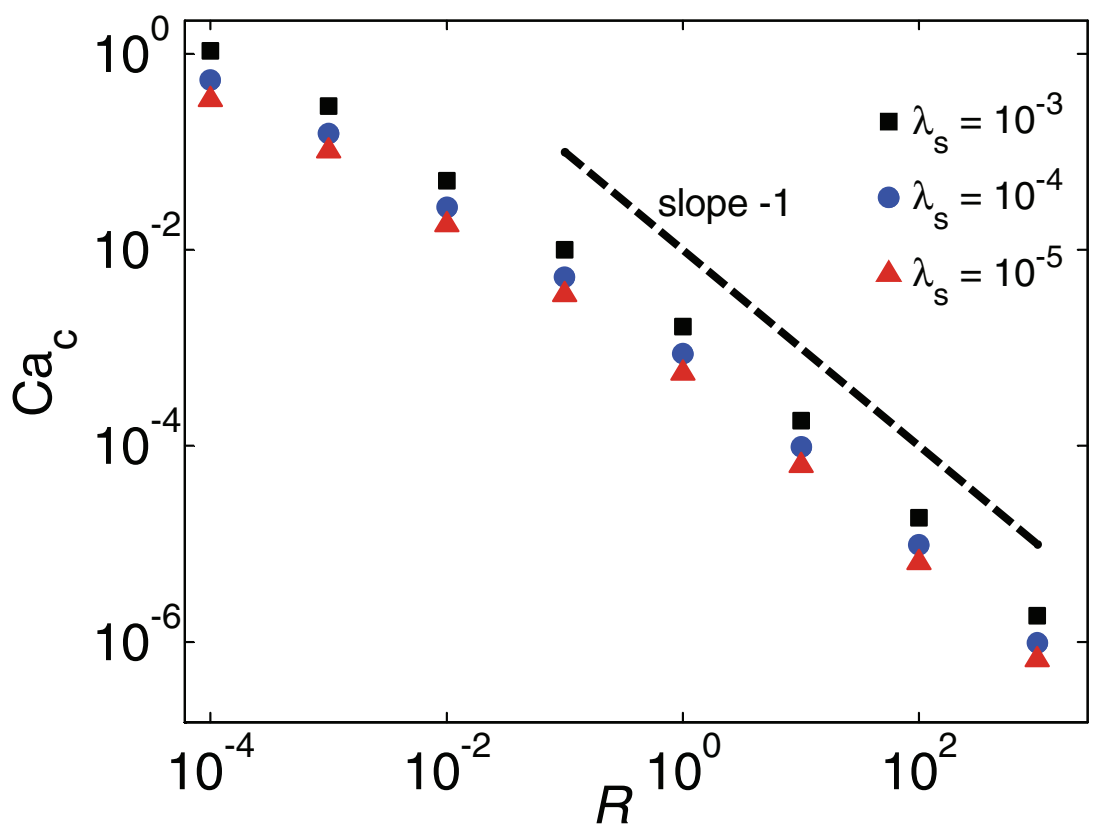

FIG. 9. Critical capillary number $\mathrm{Ca}_{\mathrm{c}}$ versus viscosity ratio $R$ for different slip lengths $\lambda_{s}\left(\theta_{e}=2.8 \mathrm{rad}\right)$. The dashed line indicates a power law with exponent -1 , which is valid for large $R$.

These observations can be interpreted as follows. As long as the viscosity of the air has a negligible effect on the flow, the curves are indistinguishable from the case $R=0$. Deviations of the $R=0$ curve signal that the air flow starts to influence the shape of the meniscus. Physically, this arises because the interface slope approaches $\pi$, leaving only a narrow wedge angle for the air flow. Figure 2 illustrates that the recirculation in the air induces significant velocity gradients: despite the small air viscosity, the stresses in the small wedge of air become comparable to those in the liquid. Mathematically, this can be derived from the function $f(\theta, R)$ as defined in (16). For small $R$ and $\theta$ close to $\pi$, we can approximate

$$
f(\theta, R) \simeq f(\theta, 0)-4 R \simeq-\frac{2(\pi-\theta)^{3}}{3 \pi}-4 R
$$

as long as $\pi-\theta \gg 2 \pi R$. For $\theta$ very close to $\pi, f(\theta, R)$ has a different asymptotic form, see the Appendix for details. The first term in (31) represents the (relative) viscous contribution inside the liquid, which vanishes in the limit $\theta \rightarrow \pi$. The second term represents the viscous contribution in the air, which will be significant once $(\pi-\theta) \sim R^{1 / 3}$. Noting that the contact line is receding from the point of view of the air phase, one understands that a critical speed appears when the effect of the air becomes important.

The dependence of the critical speed $\mathrm{Ca}_{\mathrm{c}}$ on the viscosity ratio $R$ is shown in Fig. 9 (for various slip lengths). First, we consider the limit $R \gg 1$, for which the upper fluid is actually much more viscous than the bottom fluid. This is the usual case of a receding contact line that is completely dominated by the upper (receding) phase. In this limit we expect the critical speed to scale with the viscosity of the upper phase, denoted $\eta_{g}$, such that $U_{c} \sim \gamma / \eta_{g}$. Since we have based the capillary number on the viscosity $\eta_{\ell}$, we obtain $\mathrm{Ca}_{c} \equiv U_{c} \eta_{\ell} / \gamma \sim R^{-1}$. This is indeed observed in Fig. 9 at $R \gg 1$. However, our main interest here lies in the opposite limit, i.e., $R \ll 1$, as for air entrainment. As already mentioned, the critical speed seems to increase indefinitely by reducing the viscosity ratio. This suggests that for the limiting case of $R=0$, steady menisci can be sustained at arbitrarily large plunging velocities. Our numerical resolution does not allow for a perfect determination of the asymptotics for $R \ll 1$. Enforcing a power law fit, $\mathrm{Ca}_{c} \sim R^{\beta}$, in the range $R=10^{-4}-10^{-1}$, one obtains $\beta=-0.67$. This (effective) exponent suggests that both phases play an important role in 


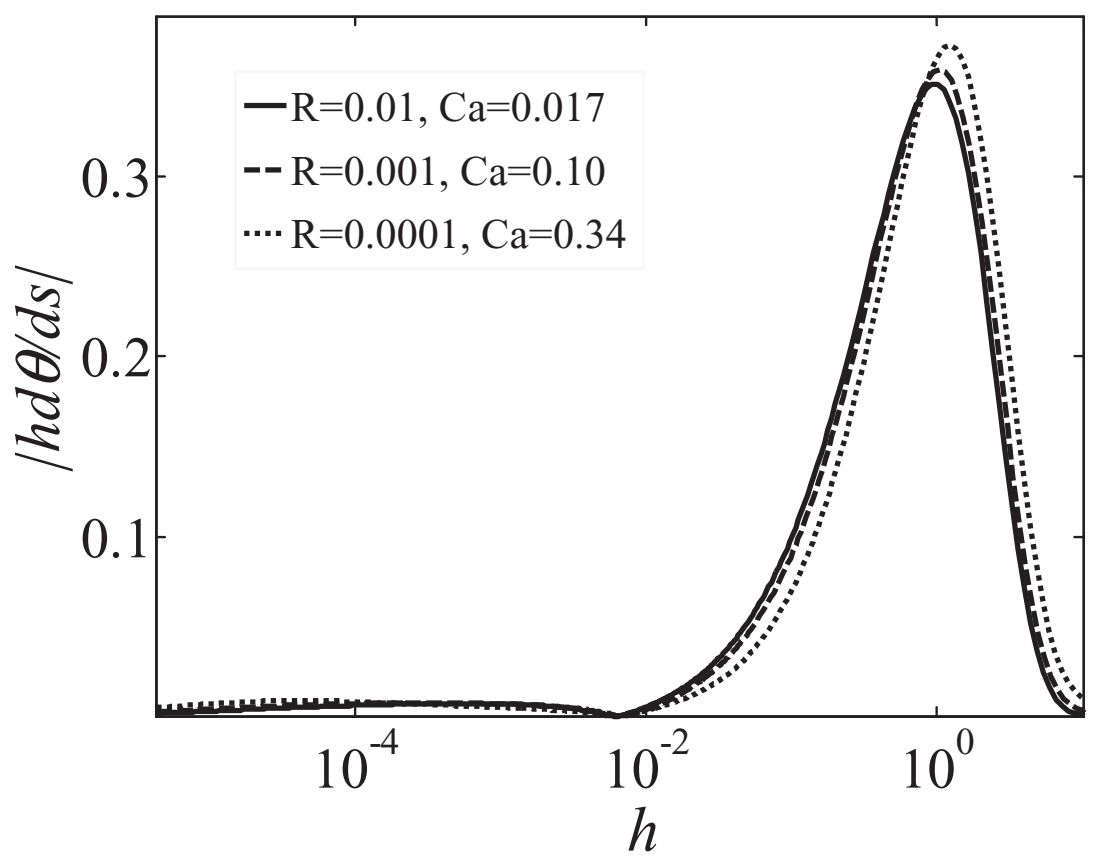

FIG. 10. Scaled curvature $|h d \theta / d s|$ vs $h$ for Ca very close to $\mathrm{Ca}_{\mathrm{c}}\left(\lambda_{s}=10^{-5}\right)$.

determining the critical speed. Namely, the exponent would be $\beta=-1$ if only $\eta_{g}$ were important, while $\beta=0$ corresponds to the case where $\eta_{\ell}$ is the only relevant viscosity.

Finally, we briefly verify the assumption of small curvature, necessary for the strict validity of the model. In Fig. 10 we plot the dimensionless curvature, $h|d \theta / d s|$, as a function of $h$ in the vicinity of the critical point $\left(R=10^{-5}, 10^{-4}\right.$, and $\left.10^{-3}, \lambda_{s}=10^{-5}\right)$. At small scales, $h|d \theta / d s| \ll 1$ for all Ca, consistent with the assumption of small curvature. However, the curvature increases significantly when approaching the bath due to the bending of interface from a large contact angle to $\pi / 2$. The magnitude is acceptable in this regime, in particular since viscous effects become less important at large scales. Inclining the plate angle to values close to $\pi$ would further reduce this bending effect, and extend the range of validity of the GL model.

\section{Dependence of the critical speed on microscopic parameters}

Apart from the viscosity ratio, the GL model contains two parameters: the slip length $\lambda_{s}$ and the microscopic (equilibrium) contact angle $\theta_{e}$. Here we discuss the dependence of $\mathrm{Ca}_{\mathrm{c}}$ on these parameters. The slip length was varied already in Fig. 9, with values $\lambda_{s}=10^{-5}, 10^{-4}$, and $10^{-3}$. As expected for wetting problems, we see a weak increase of $\mathrm{Ca}_{\mathrm{c}}$ with $\lambda_{s}$. A larger $\lambda_{s}$ reduces the range over which viscous dissipation is effective. This leads to a (logarithmic) reduction of the viscous dissipation, while the capillary driving remains unaltered. Hence, larger velocities can be achieved before air entrainment occurs.

The dependence of the critical speed on $\theta_{e}$ is investigated in Fig. 11. The figure reveals that there is no obvious universal scaling behavior for $\mathrm{Ca}_{c}$ down to viscosity ratios as small as $R=10^{-4}$. Enforcing a power-law fit, different $\theta_{e}$ would give rise to different exponents. We do clearly see that $\mathrm{Ca}_{\mathrm{c}}$ decreases with $\theta_{e}$, which is further emphasized in Fig. 12. Consistent with the results in Refs. 9 and 10 , the critical speed vanishes in the hydrophobic limit where $\theta_{e} \rightarrow \pi$. We note that for contact angles that are not close to $\pi$, the shape of the meniscus displays significant curvatures. In this sense, we expect that our results are not fully quantitative solutions of the Stokes flow problem. 


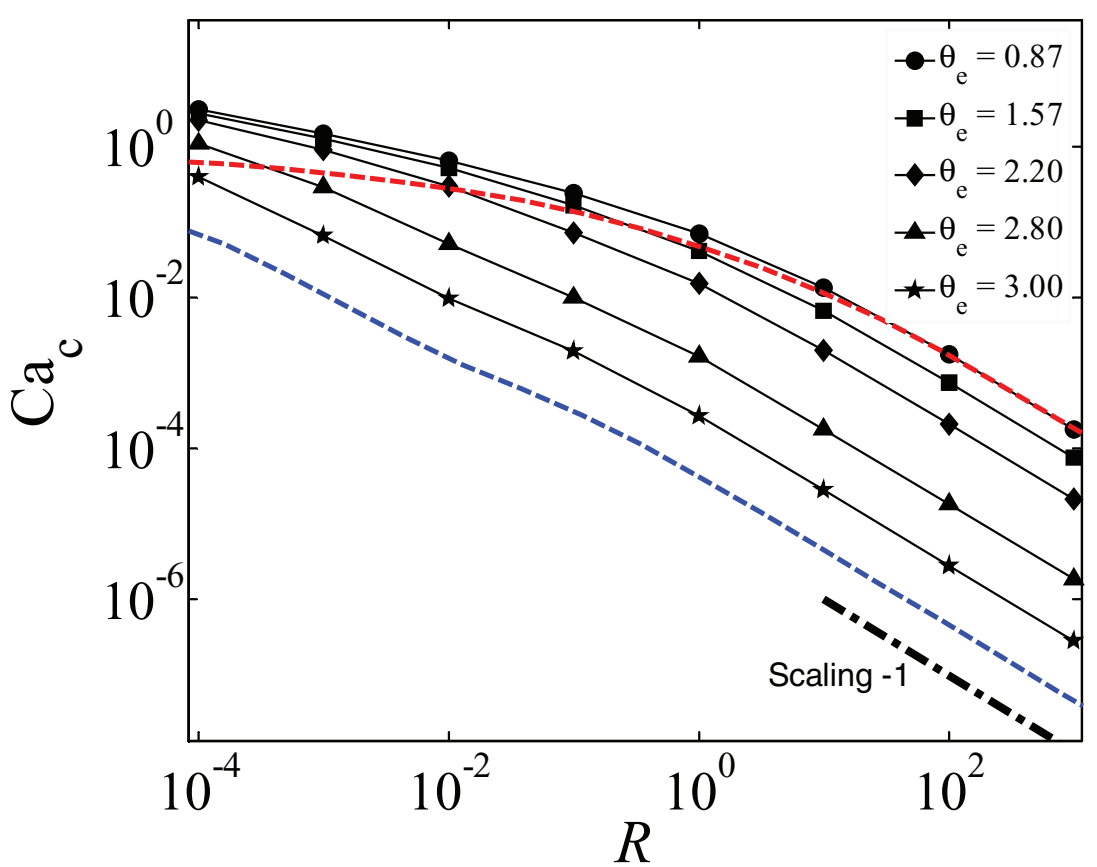

FIG. 11. Critical speed $\mathrm{Ca}_{\mathrm{c}}$ as a function of $R$ for different static contact angle $\theta_{e}\left(\lambda_{s}=0.001\right)$. Symbols are results of GL model. Dashed curves are predictions of Cox's model (top one for $\theta_{e}=0.87 \mathrm{rad}$, bottom one for $\theta_{e}=3.0 \mathrm{rad}$ ), from Eq. (8.3) of Ref. 17, for which we take the ratio between the microscopic length scale and macroscopic length scale to be $10^{-3}$ (the same value as our slip length scaled by the capillary length $\lambda_{s}$ ). The dashed-dotted line indicates slope of -1 .

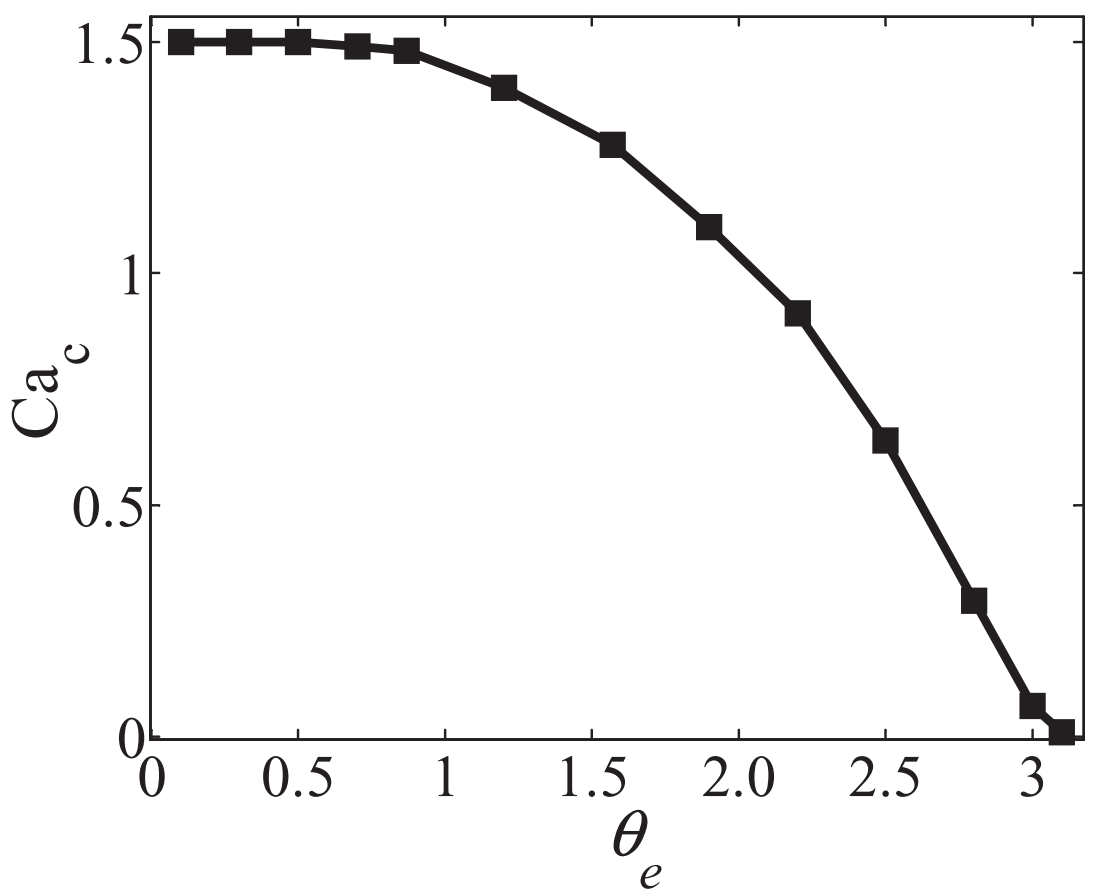

FIG. 12. Critical speed $\mathrm{Ca}_{\mathrm{c}}$ as a function of static contact angle $\theta_{e}$ for $R=0.001$ and $\lambda_{s}=0.001$. 


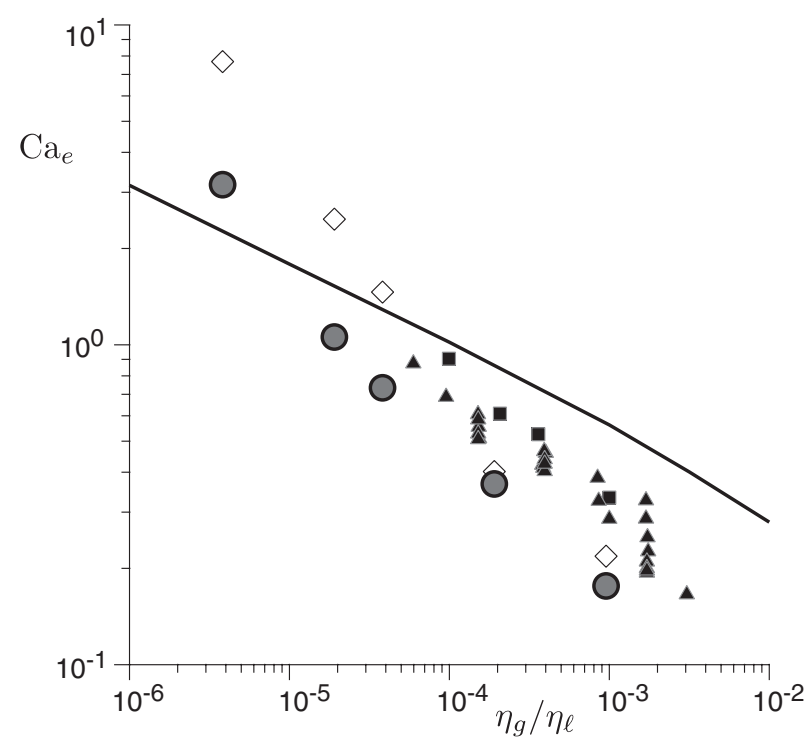

FIG. 13. Figure extracted from Ref. 8. Dimensionless entrainment (critical) speed, denoted as $\mathrm{Ca}_{e}$ versus the viscosity ratio $\eta_{g} / \eta_{\ell}$ for silicon-oil/air $(\bullet, \diamond$, data from Ref. 8), silicon-oil/air ( $\square$ data from Ref. 6), and various liquids/air ( $\boldsymbol{\Lambda}$ data from Ref. 11). Curve: Numerical results from our generalized lubrication model (slip length $\lambda_{s}=10^{-5}, \theta_{e}=50^{\circ}$ ).

\section{DISCUSSION AND CONCLUSIONS}

In this paper we present, compare and employ two distinct models to study the meniscus deformation and the onset of air entrainment in a dip-coating geometry. The first model is a generalization of the lubrication theory to a two-phase flow situation, in which a slip length is introduced to resolve the viscous singularity. The second model is a numerical one based on the discretization of the Boltzmann equation, namely, the Lattice Boltzmann method for multiphase/multicomponent fluids. In this model the viscous singularity is removed by the lattice discretization, which effectively introduces an effective slip length to the system. ${ }^{51}$ The effective slip length also depends on the choice of multiphase/multicomponent model.

The results of GL and LB have a good agreement, in particular when Ca is relatively small. When exploring larger values of $\mathrm{Ca}$, the two models start to differ as shown in Fig. 3, which can be attributed to different physics at microscopic and hydrodynamical scales (e.g., the non-zero interface thickness in the LB or strong viscous bending of interface breaks the hypothesis of small interface curvatures made in the GL approach). Yet, qualitative features, such as the bending of the meniscus and the dependence on viscosity ratio, are consistent for the two models. The transition to air entrainment for $\theta_{e}$ close to $\pi$ involves relatively weak curvatures and is thus captured by the GL model. For the LB simulations the main challenge is given by the large viscosity contrasts, which is still not fully achievable for the multiphase/multicomponent LB model used here.

In the second part of this paper, the critical speed of air entrainment is investigated by the GL model. We have found a strong dependence of critical speeds on the air viscosity, which is consistent with the experiments performed by Marchand et al. ${ }^{8}$ Remarkably, both our theoretical results and the experimental results from Ref. 8 differ from Cox's model in which $\mathrm{Ca}_{\mathrm{c}}$ is predicted to depend only logarithmically on air viscosity. ${ }^{17}$ For comparison, we have added in Fig. 11 the predictions of Cox's model, from Eq. (8.3) of Ref. 17, represented by the dashed curves (top curve for $\theta_{e}=0.87 \mathrm{rad}$, bottom one for $\theta_{e}=3.0 \mathrm{rad}$ ). For large $R$, we find that both models predict $\mathrm{Ca}_{\mathrm{c}} \mathrm{scales}$ as $R$ with scaling -1 . This regime corresponds to the usual dewetting case for which the critical speed only depends on the viscosity of the more viscous fluid. ${ }^{23,27,28}$ Note that in Cox's model, there is an undetermined factor $\epsilon$ which is defined as the ratio of the microscopic length scale to the macroscopic length scale. Here we take $\epsilon$ to be the same value as $\lambda_{s}$, which is $10^{-3}$. Interestingly, both the models predict exactly the same values of $\mathrm{Ca}_{\mathrm{c}}$ for $\theta_{e}=0.87 \mathrm{rad}$ when $R$ is large, which we consider as a coincidence since $\epsilon$ is an adjustable parameter. For $\theta_{e}=3.0 \mathrm{rad}$, Cox's and our 
results differ by a factor. More interesting things occur at small $R$, it is clearly shown in Fig. 11 that for Cox's model (red top curve ) $\mathrm{Ca}_{\mathrm{c}}$ increases extremely slowly (logarithmically) as $R$ is decreased. By contrast, our model predicts a moderate increase of $\mathrm{Ca}_{\mathrm{c}}$. Interestingly, such a weak logarithmic relation has been observed in the case of liquid impacting on liquid, ${ }^{19,20}$ for which there is no moving contact line. Both our theoretical results and experimental results from Ref. 8 therefore suggest that the mechanism leading to air entrainment can be fundamentally different depending on whether a contact line is present or not.

The GL model is directly compared with experiments in Ref. 8, see Fig. 13, and shows that the model is able to qualitatively capture the dependence of $\mathrm{Ca}_{\mathrm{c}}$ on the viscosity ratio $\eta_{g} / \eta_{\ell}$. Quantitatively, however, the agreement is not satisfactory. We believe this is due to the relatively large meniscus curvatures encountered in the experiments (static contact angle of the substrate $\approx 50^{\circ}$ ), pushing the problem beyond the assumptions of the model. It would be interesting to explore other methods to achieve a more quantitative description of air entrainment by advancing contact line, in particular for large values of $\mathrm{Ca}$. From an experimental perspective, more insight could be obtained by varying the gas viscosity or by replacing the air by a liquid of low viscosity. It would also be interesting to perform experiments with a substrate of large static contact angles.

\section{ACKNOWLEDGMENTS}

This work was partially supported by a research program of the Foundation for Fundamental Research on Matter (FOM), which is part of the Netherlands Organisation for Scientific Research (NWO). T.S.C. acknowledges financial support by the FP7 Marie Curie Initial Training Network Surface Physics for Advanced Manufacturing project ITN 215723. We are thankful for useful discussions with M. Sbragaglia. L. Biferale acknowledges support from DROEMU-FP7 IDEAS Contract No. 279004. B. Andreotti is supported by Institut Universitaire de France.

\section{APPENDIX: ASYMPTOTIC BEHAVIOR OF $f(\theta, R)$}

We discuss the behavior of the function $f(\theta, R)$ when $\theta$ is close to $\pi$ and $R$ close to zero. As presented in Sec. III A $1, f(\theta, R)$ is defined as

$$
\begin{aligned}
f(\theta, R) & \equiv \frac{2 \sin ^{3} \theta\left[R^{2} f_{1}(\theta)+2 R f_{3}(\theta)+f_{1}(\pi-\theta)\right]}{3\left[R f_{1}(\theta) f_{2}(\pi-\theta)-f_{1}(\pi-\theta) f_{2}(\theta)\right]}, \\
f_{1}(\theta) & \equiv \theta^{2}-\sin ^{2} \theta, \\
f_{2}(\theta) & \equiv \theta-\sin \theta \cos \theta, \\
f_{3}(\theta) & \equiv\left(\theta(\pi-\theta)+\sin ^{2} \theta\right) .
\end{aligned}
$$

First, we expand the terms in both the numerator and the denominator in series of $(\pi-\theta)$ and keep the leading order terms only, we end up with

$$
f(\theta, R) \simeq \frac{-2\left[\pi^{2} R^{2}+2 \pi R(\pi-\theta)+(\pi-\theta)^{4} / 3\right]}{2 \pi^{2} R+\pi(\pi-\theta)} .
$$

The asymptotic behavior of $f(\theta, R)$ depends on the relative magnitude between $(\pi-\theta)$ and $R$. For $R \ll \pi-\theta, f(\theta, R)$ can be approximated as

$$
f(\theta, R) \simeq f(\theta, 0)-4 R \simeq \frac{-2(\pi-\theta)^{3}}{3 \pi}-4 R .
$$

The contribution of air viscosity, represented by $-4 R$, will become significant once $(\pi-\theta) \sim R^{1 / 3}$.

When $\theta$ is very close to $\pi$ such that $\pi-\theta \ll R, f(\theta, R)$ goes to a different asymptotic form, i.e.,

$$
f(\theta, R) \simeq-R .
$$


If we substitute this asymptotic form of $f(\theta, R)$ into the generalized lubrication equation (22), we will see the liquid viscosity will be canceled out in the multiple CaR so that the asymptotic equation does not depend on liquid viscosity. This means in this asymptotic limit, air viscosity completely dominates the flow.

${ }^{1}$ L. D. Landau and B. V. Levich, "Dragging of a liquid by a moving plate," Acta Physicochim. USSR 17, 42 (1942).

${ }^{2}$ B. V. Deryaguin, "On the thickness of a layer of liquid remaining on the walls of vessels after their emptying, and the theory of the application of photoemulsion after coating on the cine film," Acta Physicochim. USSR 20, 349 (1943).

${ }^{3}$ F. P. Bretherton, "The motion of long bubbles in tubes," J. Fluid Mech. 10, 166 (1961).

${ }^{4}$ D. Bonn, J. Eggers, J. Indekeu, J. Meunier, and E. Rolley, "Wetting and spreading," Rev. Mod. Phys. 81, 739 (2009).

${ }^{5}$ J. H. Snoeijer and B. Andreotti, "Moving contact lines: Scales, regimes, and dynamical transitions," Annu. Rev. Fluid Mech. 45, 269 (2013).

${ }^{6}$ H. Benkreira and M. I. Khan, "Air entrainment in dip coating under reduced air pressures," Chem. Eng. Sci. 63, 448-459 (2008).

${ }^{7}$ H. Benkreira and J. B. Ikin, "Dynamic wetting and gas viscosity effects," Chem. Eng. Sci. 65, 1790-1796 (2010).

${ }^{8}$ A. Marchand, T. S. Chan, J. H. Snoeijer, and B. Andreotti, "Air entrainment by contact lines of a solid plate plunged into a viscous fluid," Phys. Rev. Lett. 108, 204501 (2012).

${ }^{9}$ C. Duez, C. Ybert, C. Clanet, and L. Bocquet, "Making a splash with water repellency," Nat. Phys. 3, 180 (2007).

${ }^{10}$ R. Ledesma-Aguilar, R. Nistal, A. Hernández-Machado, and I. Pagonabarraga, "Controlled drop emission by wetting properties in driven liquid filaments," Nature Mater. 10,367-371 (2011).

${ }^{11}$ R. Burley and B. S. Kennedy, "A study of the dynamic wetting behavior of polyester tapes," Br. Polym. J. 8, 140-143 (1976).

${ }^{12}$ O. Cohu and H. Benkreira, "Entrainment of air by a solid surface plunging into a non- newtonian liquid," AIChE J. 44(11), 2360-2368 (1998).

${ }^{13}$ O. Cohu and H. Benkreira, "Air entrainment in angled dip coating," Chem. Eng. Sci. 53, 533 (1998).

${ }^{14}$ M. T. Ghannam and M. N. Esmail, "Effect of substrate entry angle on air entrainment in liquid coating," AIChE J. 36(8), 1283-1286 (1990).

${ }^{15}$ P. G. Simpkins and V. J. Kuck, "On air entrainment in coatings," J. Colloid Interface Sci. 263, 562 (2003).

${ }^{16}$ Y. C. Severtson and C. Aidun, "Stability of two-layer stratified flow in inclined channels: Applications to air entrainment in coating systems," J. Fluid Mech. 312, 173 (1996).

${ }^{17}$ R. G. Cox, "The dynamics of the spreading of liquids on a solid surface. Part 1. Viscous flow," J. Fluid Mech. 168, 169-194 (1986).

${ }^{18}$ B. Bolton and S. Middleman, “Air entrainment in a roll coating system,” Chem. Eng. Sci. 35, 597 (1980).

${ }^{19}$ J. Eggers, "Air entrainment through free-surface cusps," Phys. Rev. Lett. 86, 4290 (2001).

${ }^{20}$ É. Lorenceau, F. Restagno, and D. Quéré, "Fracture of a viscous liquid," Phys. Rev. Lett. 90, 184501 (2003).

${ }^{21}$ M. Sbragaglia, K. Sugiyama, and L. Biferale, "Wetting failure and contact line dynamics in a Couette flow," J. Fluid Mech. 614, 471 (2008).

${ }^{22}$ T. D. Blake and K. J. Ruschak, "Wetting: Static and dynamic contact lines," in Liquid Film Coating, edited by S. F. Kistler and P. M. Schweizer (Chapman and Hall, London, 1997).

${ }^{23}$ J. Eggers, "Hydrodynamic theory of forced dewetting," Phys. Rev. Lett. 93, 094502 (2004).

${ }^{24}$ R. V. Sedev and J. G. Petrov, "The critical condition for transition from steady wetting to film entrainment," Colloids Surf. 53, 147 (1991).

${ }^{25}$ D. Quéré, "On the minimal velocity of forced spreading in partial wetting (in French)," C. R. Acad. Sci. Paris, Ser. II 313, 313-318 (1991).

${ }^{26}$ J. H. Snoeijer, G. Delon, M. Fermigier, and B. Andreotti, “Avoided critical behavior in dynamically forced wetting,” Phys. Rev. Lett. 96, 174504 (2006).

${ }^{27}$ J. H. Snoeijer, B. Andreotti, G. Delon, and M. Fermigier, "Relaxation of a dewetting contact line. Part 1: A full-scale hydrodynamic calculation," J. Fluid Mech. 579, 63 (2007).

${ }^{28}$ G. Delon, M. Fermigier, J. H. Snoeijer, and B. Andreotti, "Relaxation of a dewetting contact line. Part 2: Experiments," J. Fluid Mech. 604, 55 (2008).

${ }^{29}$ T. D. Blake and K. J. Ruschak, “A maximum speed of wetting,” Nature (London) 282, 489-491 (1979).

${ }^{30}$ R. Burley and B. S. Kennedy, "An experimental study of air entrainment at a solid/liquid/gas interface," Chem. Eng. Sci. 31, 901-911 (1976).

${ }^{31}$ P. G. Simpkins and V. J. Kuck, "Air entrapment in coatings by way of a tip-streaming meniscus," Nature (London) 403, 641-643 (2000).

${ }^{32}$ C. Huh and L. E. Scriven, "Hydrodynamic model of steady movement of a solid/liquid/fluid contact line," J. Colloid Interface Sci. 35, 85-101 (1971).

${ }^{33}$ A. Oron, S. H. Davis, and S. G. Bankoff, "Long-scale evolution of thin liquid films," Rev. Mod. Phys. 69, 931-980 (1997).

${ }^{34}$ J. H. Snoeijer, "Free surface flows with large slopes: Beyond lubrication theory," Phys. Fluids 18, 021701 (2006).

${ }^{35}$ O. V. Voinov, "Hydrodynamics of wetting," Fluid Dyn. 11, 714-721 (1976).

${ }^{36}$ J. Eggers, "Toward a description of contact line motion at higher capillary numbers," Phys. Fluids 16, 3491 (2004).

${ }^{37}$ R. Buckingham, M. Shearer, and A. Bertozzi, "Thin film traveling waves and the Navier slip condition," SIAM J. Appl. Math. 63, 722-744 (2003).

${ }^{38}$ P. L. Bhatnagar, E. P. Gross, and M. Krook, "A model for collision processes in gases. I. Small amplitude processes in charged and neutral one-component systems," Phys. Rev. 94, 511-525 (1954).

${ }^{39} \mathrm{~S}$. Succi, The Lattice Boltzmann Equation for Fluid Dynamics and Beyond, Numerical Mathematics and Scientific Computation (Clarendon Press, Oxford, 2001). 
${ }^{40}$ D. A. Wolf-Gladrow, Lattice Gas Cellular Automata and Lattice Boltzmann Models, Lecture Notes in Mathematics Vol. 1725 (Springer, Berlin/Heidelberg, 2005).

${ }^{41}$ X. Shan and G. Doolen, "Multicomponent lattice-Boltzmann model with interparticle interaction,” J. Stat. Phys. 81, 379-393 (1995).

${ }^{42}$ X. Shan and H. Chen, "Lattice Boltzmann model for simulating flows with multiple phases and components," Phys. Rev. E 47, 1815 (1993).

${ }^{43} \mathrm{X}$. Shan, "Simulation of nonideal gases and liquid-gas phase transitions by the lattice Boltzmann equation," Phys. Rev. E 49, 2941-2948 (1994).

${ }^{44}$ R. Benzi, L. Biferale, M. Sbragaglia, S. Succi, and F. Toschi, "Mesoscopic modeling of a two-phase flow in the presence of boundaries: The contact angle," Phys. Rev. E 74, 021509 (2006).

${ }^{45}$ H. Huang, Jr., D. T. Thorne, M. G. Schaap, and M. C. Sukop, "Proposed approximation for contact angles in Shan-andChen-type multicomponent multiphase lattice Boltzmann models," Phys. Rev. E 76, 066701 (2007)

${ }^{46} \mathrm{E}$. Rame and S. Garoff, "Microscopic and macroscopic dynamic interface shapes and the interpretation of dynamic contact angles," J. Colloid Interface Sci. 177, 234-244 (1996).

${ }^{47}$ J. Ziegler, J. H. Snoeijer, and J. Eggers, "Film transitions of receding contact lines," Eur. Phys. J. Spec. Top. 166, 177 (2009).

${ }^{48}$ J. H. Snoeijer, J. Ziegler, B. Andreotti, M. Fermigier, and J. Eggers, "Thick films coating a plate withdrawn from a bath," Phys. Rev. Lett. 100, 244502 (2008).

${ }^{49}$ T. S. Chan, T. Gueudré, and J. H. Snoeijer, "Maximum speed of dewetting on a fiber," Phys. Fluids 23, 112103 (2011).

${ }^{50}$ T. S. Chan, J. H. Snoeijer, and J. Eggers, "Theory of the forced wetting transition," Phys. Fluids 24, 072104 (2012).

${ }^{51}$ S. Srivastava, P. Perlekar, L. Biferale, M. Sbragaglia, J. H. M. ten Thije Boonkkamp, and F. Toschi, "A study of fluid interfaces and moving contact lines using the lattice Boltzmann method," Comm. Comp. Phys. 13, 725-740 (2013). 